\title{
Sufficiency and Wolfe Type Duality for Nonsmooth Multiobjective Programming Problems
}

\author{
Gang An1, Xiaoyan Gao² \\ ${ }^{1}$ College of Science, Xi'an Shiyou University, Xi'an, China \\ ${ }^{2}$ College of Science, Xi'an University of Science and Technology, Xi'an, China \\ Email: 1666465514@qq.com
}

How to cite this paper: An, G. and Ga, X.Y. (2018) Sufficiency and Wolfe Type Duality for Nonsmooth Multiobjective Programming Problems. Advances in Pure Mathematics, 8, 755-763. https://doi.org/10.4236/apm.2018.88045

Received: July 30, 2018

Accepted: August 17, 2018

Published: August 20, 2018

Copyright (c) 2018 by authors and Scientific Research Publishing Inc. This work is licensed under the Creative Commons Attribution International License (CC BY 4.0).

http://creativecommons.org/licenses/by/4.0/

\begin{abstract}
In this paper, a class of nonsmooth multiobjective programming problems is considered. We introduce the new concept of invex of order $\sigma(B, \varphi)-V-$ type II for nondifferentiable locally Lipschitz functions using the tools of Clarke subdifferential. The new functions are used to derive the sufficient optimality condition for a class of nonsmooth multiobjective programming problems. Utilizing the sufficient optimality conditions, weak and strong duality theorems are established for Wolfe type duality model.
\end{abstract}

\section{Keywords}

Multiobjective Programming, Optimality Condition, Locally Lipschitz Function, Wolfe Type Dual Problem

\section{Introduction}

The field of multiobjective programming, also called vector programming, has grown remarkably in different directions in the settings of optimality conditions and duality theory since the 1980s. It has been enriched by the applications of various types of generalizations of convexity theory, with and without differentiability assumptions. The Clarke subdifferential [1] (also called the Clarke generalized gradient) is an important tool to derive optimality conditions for nonsmooth optimization problems. Together with the Clarke's subdifferential, many generalized convexity or invexity functions were generalized to locally Lipschitz functions. Based upon the generalized functions, several sufficient optimality conditions and duality results were established for the optimization problems. We can see for examples [2]-[8]. In [9] Upadhyay introduced some 
new generalizations of the concept of $(\phi, \rho)$-invexity and established the necessary and sufficient optimality conditions for a class of nonsmooth semi-infinite minmax programming problems. In [10] the new concepts of $(\phi, \rho)-V$-type I were introduced. Sufficient optimality conditions and Mond-Weir duality results were obtained for nonsmooth multiobjective programming problems. Recently, many researchers have been interested in other types of solution concepts. One of them is higher order strict minimizer. In [11] and [12] some sufficient conditions and duality results were obtained for the new concept of strict minimizer of higher order for a multiobjective optimization problem.

In this paper, we consider the nonsmooth multiobjective programming including the locally Lipschitz functions. The new concepts of invex of order $\sigma(B, \varphi)-V$-type II functions are introduced. Then, a sufficient optimality condition is obtained for the nondifferentiable multiobjective programming problem under the new functions and the Wolfe type duality results are obtained.

\section{Preliminaries and Definitions}

Let $R^{n}$ be the $n$-dimensional Euclidean space and let $X$ be a nonempty open subset of $R^{n}$. For $x=\left(x_{1}, x_{2}, \cdots, x_{n}\right)^{\mathrm{T}}, y=\left(y_{1}, y_{2}, \cdots, y_{n}\right)^{\mathrm{T}} \in R^{n}$, we denote:

$$
\begin{aligned}
& x=y \Leftrightarrow x_{i}=y_{i}, \quad i=1,2, \cdots, n ; \\
& x \leqq y \Leftrightarrow x_{i} \leq y_{i}, \quad i=1,2, \cdots, n ; \\
& x \leq y \Leftrightarrow x_{i} \leqq y_{i}, \quad i=1,2, \cdots, n \text { and } x \neq y ; \\
& x<y \Leftrightarrow x_{i}<y_{i}, \quad i=1,2, \cdots, n ; \\
& x \in R_{+}^{n} \Leftrightarrow x \geqq 0 .
\end{aligned}
$$

Definition 2.1. [1] The function $f: X \rightarrow R$ is said to be locally Lipschitz at $x \in X$, if there exist scalars $k>0$ and $\varepsilon>0$, such that

$$
|f(y)-f(z)| \leqq k\|y-z\|, \text { for all } y, z \in B(x, \varepsilon) .
$$

where $B(x, \varepsilon)$ is the open ball of radius $\varepsilon$ about $x$.

Definition 2.2. [1] The generalized directional derivative of a locally Lipschitz function $f$ at $x$ in the direction $d$, denoted by $f^{0}(x ; d)$, is as follows:

$$
f^{0}(x ; d)=\lim _{y \rightarrow x} \sup _{\lambda \rightarrow 0^{+}} \frac{f(y+\lambda d)-f(y)}{\lambda} .
$$

Definition 2.3. [1] The generalized gradient of $f: X \rightarrow R$ at $x \in X$, denoted by $\partial f(x)$, is defined as follows:

$$
\partial f(x)=\left\{\xi \in R^{n}: f^{0}(x ; d) \geq\langle\xi, d\rangle, \forall d \in R^{n}\right\} .
$$

where $\langle\cdot, \cdot\rangle$ is the inner product in $R^{n}$.

Consider the following nonsmooth multiobjective programming problem:

$$
\begin{gathered}
\text { Minimize } \\
\text { s.t. } \\
g_{j}(x)=\left(f_{1}(x), f_{2}(x), \cdots, j=1,2, \cdots, m,\right. \\
x \in X .
\end{gathered}
$$


where $f_{i}: X \rightarrow R(i \in K=\{1,2, \cdots, k\}) \quad$ and $\quad g_{j}: X \rightarrow R(j \in M=\{1,2, \cdots, m\})$ are locally Lipschitz functions and $X$ is a convex set in $R^{n}$.

Let $X_{0}=\left\{x \mid g_{j}(x) \leqq 0, j \in M\right\}$ be the set of feasible solutions of (MP), and

$$
J(x)=\left\{j \mid g_{j}(x)=0, x \in X_{0}, j \in M\right\} .
$$

Definition 2.4. A point $\bar{x} \in X_{0}$ is a strict minimizer of order $\sigma$ for (MP) with respect to a nonlinear function $\psi: X \times X \rightarrow R^{n}$, if for a constant $\rho \in \operatorname{int} R_{+}^{k}$, such that

$$
f(\bar{x}) \leqq f(x)+\rho\|\psi(x, \bar{x})\|^{\sigma}, \text { for all } x \in X_{0} .
$$

Throughout the paper, we suppose that $\eta: X \times X \rightarrow R^{n} ; b_{0}, b_{1}: X \times X \rightarrow R_{+}$; $\varphi_{0}, \varphi_{1}: R \rightarrow R ; \alpha, \beta: X \times X \rightarrow R_{+} \backslash\{0\} ; \rho_{i}, \tau \in R_{+}, i \in K$.

Definition 2.5. $(f, g)$ is said to be invex of order $\sigma(B, \varphi)-V$-type II at $\bar{x} \in X$, if there exist $\eta, b_{0}, b_{1}, \varphi_{0}, \varphi_{1}, \alpha, \beta, \rho_{i}(i \in K), \tau$ and some vectors $\lambda \in R_{+}^{k}$ and $\mu \in R_{+}^{m}$ such that for all $x \in X$ the following inequalities hold:

$$
\begin{gathered}
b_{0}(x, \bar{x}) \varphi_{0}\left[\sum_{i=1}^{k} \lambda_{i}\left(f_{i}(x)-f_{i}(\bar{x})+\rho_{i}\|\psi(x, \bar{x})\|^{\sigma}\right)\right] \\
\geqq \alpha(x, \bar{x})\left\langle\sum_{i=1}^{k} \lambda_{i} \xi_{i}, \eta(x, \bar{x})\right\rangle, \forall \xi_{i} \in \partial f_{i}(\bar{x}), i \in K \\
-b_{1}(x, \bar{x}) \varphi_{1}\left[\sum_{j=1}^{m} \mu_{j} g_{j}(\bar{x})\right] \geqq \beta(x, \bar{x})\left\langle\sum_{j=1}^{m} \mu_{j} \zeta_{j}, \eta(x, \bar{x})\right\rangle+\tau\|\psi(x, \bar{x})\|^{\sigma}, \\
\forall \zeta_{j} \in \partial g_{j}(\bar{x}), j \in M .
\end{gathered}
$$

Definition 2.6. $(f, g)$ is said to be (pseudo, quasi) invex of order $\sigma(B, \varphi)-V$-type II at $\bar{x} \in X$, if there exist $\eta, b_{0}, b_{1}, \varphi_{0}, \varphi_{1}, \alpha, \beta, \rho_{i}(i \in K), \tau$ and some vectors $\lambda \in R_{+}^{k}$ and $\mu \in R_{+}^{m}$ such that for all $x \in X$ the following inequalities hold:

$$
\begin{gathered}
\alpha(x, \bar{x})\left\langle\sum_{i=1}^{k} \bar{\lambda}_{i} \xi_{i}, \eta(x, \bar{x})\right\rangle \geqq 0, \forall \xi_{i} \in \partial f_{i}(\bar{x}), i \in K \\
\Rightarrow b_{0}(x, \bar{x}) \varphi_{0}\left[\sum_{i=1}^{k} \lambda_{i}\left(f_{i}(x)-f_{i}(\bar{x})+\rho_{i}\|\psi(x, \bar{x})\|^{\sigma}\right)\right] \geqq 0, \\
-b_{1}(x, \bar{x}) \varphi_{1}\left[\sum_{j=1}^{m} \mu_{j} g_{j}(\bar{x})\right] \leqq 0 \\
\Rightarrow \beta(x, \bar{x})\left\langle\sum_{j=1}^{m} \mu_{j} \zeta_{j}, \eta(x, \bar{x})\right\rangle+\tau\|\psi(x, \bar{x})\|^{\sigma} \leqq 0, \forall \zeta_{j} \in \partial g_{j}(\bar{x}), j \in M .
\end{gathered}
$$

\section{Optimality Condition}

In this section, we establish sufficient optimality conditions for a strict minimizer of (MP).

Theorem 3.1. Let $\bar{x} \in X_{0}$. Suppose that

1) There exist $\lambda_{i} \geqq 0, i \in K, \quad \sum_{i=1}^{k} \lambda_{i}=1, \mu_{j} \geqq 0, j \in J(\bar{x})$, such that 


$$
0 \in \sum_{i=1}^{k} \lambda_{i} \partial f_{i}(\bar{x})+\sum_{j \in J(\bar{x})} \mu_{j} \partial g_{j}(\bar{x})
$$

2) $\left(f, g_{J}\right)$ is invex of order $\sigma(B, \varphi)-V$-type II at $\bar{x}$,

3) $b_{0}(x, \bar{x})>0, b_{1}(x, \bar{x}) \geqq 0 ; a<0 \Rightarrow \varphi_{0}(a)<0, a=0 \Rightarrow \varphi_{1}(a)=0$.

Then $\bar{x}$ is a strict minimizer of order $\sigma$ for (MP).

Proof: Since $0 \in \sum_{i=1}^{k} \lambda_{i} \partial f_{i}(\bar{x})+\sum_{j \in J(\bar{x})} \mu_{j} \partial g_{j}(\bar{x})$, there exists $\xi_{i} \in \partial f_{i}(\bar{x}), i \in K$, $\zeta_{j} \in \partial g_{j}(\bar{x}), j \in J(\bar{x})$, such that

$$
\sum_{i=1}^{k} \lambda_{i} \xi_{i}+\sum_{j \in J(\bar{x})} \mu_{j} \zeta_{j}=0
$$

whence

$$
\left\langle\sum_{i=1}^{k} \lambda_{i} \xi_{i}+\sum_{j \in J(\bar{x})} \mu_{j} \zeta_{j}, \eta(x, \bar{x})\right\rangle=0
$$

Suppose that $\bar{x}$ is not a strict minimizer of order $\sigma$ for (MP). Then there exists $x \in X_{0}$ and $\rho \in R_{+}^{k}$, such that

$$
f(\bar{x})>f(x)+\rho\|\psi(x, \bar{x})\|^{\sigma} .
$$

By $\lambda_{i} \geqq 0, i \in K, \sum_{i=1}^{k} \lambda_{i}=1$ and hypothesis 3 ), we have

$$
b_{0}(x, \bar{x}) \varphi_{0}\left[\sum_{i=1}^{k} \lambda_{i}\left(f_{i}(x)-f_{i}(\bar{x})+\rho_{i}\|\psi(x, \bar{x})\|^{\sigma}\right)\right]<0 .
$$

Since $g_{j}(\bar{x})=0, j \in J(\bar{x})$ and $\mu_{j} \geqq 0, j \in J(\bar{x})$, and hypothesis 3 ), we get

$$
-b_{1}(x, \bar{x}) \varphi_{1}\left[\sum_{j \in J(\bar{x})} \mu_{j} g_{j}(\bar{x})\right]=0 \text {. }
$$

In view of the hypothesis 1), one finds from (12) and (13) that

$$
\begin{gathered}
\alpha(x, \bar{x})\left\langle\sum_{i=1}^{k} \lambda_{i} \xi_{i}, \eta(x, \bar{x})\right\rangle<0, \forall \xi_{i} \in \partial f_{i}(\bar{x}), i \in K . \\
\beta(x, \bar{x})\left\langle\sum_{j \in J(\bar{x})} \mu_{j} \zeta_{j}, \eta(x, \bar{x})\right\rangle+\tau\|\psi(x, \bar{x})\|^{\sigma} \leqq 0, \forall \zeta_{j} \in \partial g_{j}(\bar{x}), j \in J(\bar{x}) .
\end{gathered}
$$

From $\alpha(x, \bar{x})>0, \beta(x, \bar{x})>0$ and $\tau \geqq 0$, we obtain

$$
\begin{gathered}
\left\langle\sum_{i=1}^{k} \lambda_{i} \xi_{i}, \eta(x, \bar{x})\right\rangle<0, \forall \xi_{i} \in \partial f_{i}(\bar{x}), i \in K . \\
\left\langle\sum_{j \in J(\bar{x})} \mu_{j} \zeta_{j}, \eta(x, \bar{x})\right\rangle \leqq 0, \forall \zeta_{j} \in \partial g_{j}(\bar{x}), j \in J(\bar{x}) .
\end{gathered}
$$

Also

$$
\left\langle\sum_{i=1}^{k} \lambda_{i} \xi_{i}+\sum_{j \in J(\bar{x})} \mu_{j} \zeta_{j}, \eta(x, \bar{x})\right\rangle<0, \forall \xi_{i} \in \partial f_{i}(\bar{x}), i \in K, \forall \zeta_{j} \in \partial g_{j}(\bar{x}), j \in J(\bar{x})
$$


which contradicts (10). Hence the result is true.

\section{Wolfe Type Duality}

In this section, we consider the Wolfe type dual for the primal problem (MP) and establish various duality theorems. Let e be the vector of $R^{k}$ whose components are all ones.

(MD)

$$
\begin{aligned}
& \text { Maximize } F(u)=f(u)+\bar{\mu}^{\mathrm{T}} g(u) e \\
& =\left(f_{1}(u)+\sum_{j=1}^{m} \bar{\mu}_{j} g_{j}(u), \cdots, f_{k}(u)+\sum_{j=1}^{m} \bar{\mu}_{j} g_{j}(u)\right) \\
& \text { subject to } 0 \in \sum_{i=1}^{k} \bar{\lambda}_{i} \partial f_{i}(u)+\sum_{j=1}^{m} \bar{\mu}_{j} \partial g_{j}(u) \text {, } \\
& \bar{\lambda}_{i} \geqq 0, \sum_{i=1}^{k} \bar{\lambda}_{i}=1, \quad i \in K, \\
& \bar{\mu}_{j} \geqq 0, j \in M \text {. }
\end{aligned}
$$

Let

$U=\left\{(u, \bar{\lambda}, \bar{\mu}) \in X \times R_{+}^{k} \times R_{+}^{m} \mid 0 \in \sum_{i=1}^{k} \bar{\lambda}_{i} \partial f_{i}(u)+\sum_{j=1}^{m} \bar{\mu}_{j} \partial g_{j}(u), \bar{\lambda} \geq 0, \sum_{i=1}^{k} \bar{\lambda}_{i}=1, \bar{\mu} \geqq 0\right\}$ be the set of all feasible solutions in problem (MD).

Theorem 4.1. (weak duality) Let $\forall x \in X_{0}$ and $\forall(u, \bar{\lambda}, \bar{\mu}) \in W$ be feasible solutions for (MP) and (MD), respectively. Moreover, assume that

1) $(f, g)$ is invex of order $\sigma(B, \varphi)-V$-type II at $u$,

2) $b_{1}(x, u)>b_{0}(x, u)>0 ; a<b \Rightarrow \varphi_{0}(a)<\varphi_{1}(b) ; \alpha(x, u)=\beta(x, u)$.

Then the following can hold:

$$
f(x) \geqq F(u)-\rho\|\psi(x, u)\|^{\sigma} .
$$

Proof: Suppose contrary to the result that $f(x)<F(u)-\rho\|\psi(x, u)\|^{\sigma}$ holds, then we have

$$
f_{i}(x)<f_{i}(u)+\sum_{j=1}^{m} \bar{\mu}_{j} g_{j}(u)-\rho_{i}\|\psi(x, u)\|^{\sigma}, i \in K .
$$

which implies

$$
f_{i}(x)-f_{i}(u)+\rho_{i}\|\psi(x, u)\|^{\sigma}<\sum_{j=1}^{m} \bar{\mu}_{j} g_{j}(u), i \in K
$$

Using $\quad \bar{\lambda}_{i} \geqq 0, i \in K, \sum_{i=1}^{k} \bar{\lambda}_{i}=1$, we have

$$
\sum_{i=1}^{k} \bar{\lambda}_{i}\left(f_{i}(x)-f_{i}(u)+\rho_{i}\|\psi(x, \bar{x})\|^{\sigma}\right)<\sum_{j=1}^{m} \bar{\mu}_{j} g_{j}(u) .
$$

By hypothesis 2), we have

$$
b_{0}(x, u) \varphi_{0}\left[\sum_{i=1}^{k} \bar{\lambda}_{i}\left(f_{i}(x)-f_{i}(u)+\rho_{i}\|\psi(x, u)\|^{\sigma}\right)\right]<b_{1}(x, u) \varphi_{1}\left[\sum_{j=1}^{m} \bar{\mu}_{j} g_{j}(u)\right] \text {. }
$$

with hypothesis 1) and 2), the above inequality yields 


$$
\begin{aligned}
& \alpha(x, u)\left\langle\sum_{i=1}^{k} \bar{\lambda}_{i} \xi_{i}, \eta(x, u)\right\rangle<-\alpha(x, u)\left\langle\sum_{j=1}^{m} \bar{\mu}_{j} \zeta_{j}, \eta(x, u)\right\rangle-\tau\|\psi(x, u)\|^{\sigma}, \\
& \forall \xi_{i} \in \partial f_{i}(u), i \in K, \forall \zeta_{j} \in \partial g_{j}(u), j \in M .
\end{aligned}
$$

That is

$$
\begin{aligned}
& \left\langle\sum_{i=1}^{k} \bar{\lambda}_{i} \xi_{i}+\sum_{j=1}^{m} \bar{\mu}_{j} \zeta_{j}, \eta(x, u)\right\rangle<-\frac{\tau}{\alpha(x, u)}\|\psi(x, u)\|^{\sigma}, \\
& \forall \xi_{i} \in \partial f_{i}(u), i \in K, \forall \zeta_{j} \in \partial g_{j}(u), j \in M .
\end{aligned}
$$

From $\tau \geqq 0$, which implies

$$
\left\langle\sum_{i=1}^{k} \bar{\lambda}_{i} \xi_{i}+\sum_{j=1}^{m} \bar{\mu}_{j} \zeta_{j}, \eta(x, u)\right\rangle<0, \forall \xi_{i} \in \partial f_{i}(u), i \in K, \forall \zeta_{j} \in \partial g_{j}(u), j \in M
$$

On the other hand, by using the constraint conditions of (MD), there exist

$$
\xi_{i} \in \partial f_{i}(u), i \in K \text { and } \zeta_{j} \in \partial g_{j}(u), j \in M,
$$

such that

$$
\sum_{i=1}^{k} \bar{\lambda}_{i} \xi_{i}+\sum_{j=1}^{m} \bar{\mu}_{j} \zeta_{j}=0
$$

Also,

$$
\left\langle\sum_{i=1}^{k} \bar{\lambda}_{i} \xi_{i}+\sum_{j=1}^{m} \bar{\mu}_{j} \zeta_{j}, \eta(x, u)\right\rangle=0 .
$$

which contradicts (26). Then the result is true.

Theorem 4.2. (weak duality) Let $\forall x \in X_{0}$ and $\forall(u, \bar{\lambda}, \bar{\mu}) \in W$ be feasible solutions for (MP) and (MD), respectively. Moreover, assume that

1) $(f, g)$ is (pseudo,quasi) invex of order $\sigma(B, \varphi)-V$-type II at $u$,

2) $b_{1}(x, u)>b_{0}(x, u)>0 ; a<b \Rightarrow \varphi_{0}(a)<\varphi_{1}(b)=0$.

Then the following can hold:

$$
f(x) \geqq F(u)-\rho\|\psi(x, u)\|^{\sigma} .
$$

Proof: Suppose contrary to the result that $f(x)<F(u)-\rho\|\psi(x, u)\|^{\sigma}$ holds, then we have

$$
f_{i}(x)<f_{i}(u)+\sum_{j=1}^{m} \bar{\mu}_{j} g_{j}(u)-\rho_{i}\|\psi(x, u)\|^{\sigma}, i \in K
$$

Also

$$
f_{i}(x)-f_{i}(u)+\rho_{i}\|\psi(x, u)\|^{\sigma}<\sum_{j=1}^{m} \bar{\mu}_{j} g_{i}(u), i \in K .
$$

Since $\bar{\lambda}_{i} \geqq 0, i \in K, \sum_{i=1}^{k} \bar{\lambda}_{i}=1$, which yields

$$
\sum_{i=1}^{k} \bar{\lambda}_{i}\left(f_{i}(x)-f_{i}(u)+\rho_{i}\|\psi(x, \bar{x})\|^{\sigma}\right)<\sum_{j=1}^{m} \bar{\mu}_{j} g_{j}(u) .
$$

It follows from hypothesis 2) that 
$b_{0}(x, u) \varphi_{0}\left[\sum_{i=1}^{k} \bar{\lambda}_{i}\left(f_{i}(x)-f_{i}(u)+\rho_{i}\|\psi(x, u)\|^{\sigma}\right)\right]<b_{1}(x, u) \varphi_{1}\left[\sum_{j=1}^{m} \bar{\mu}_{j} g_{j}(u)\right]=0$.

In the view of hypothesis 1), one finds from (33) that

$$
\alpha(x, u)\left\langle\sum_{i=1}^{k} \bar{\lambda}_{i} \xi_{i}, \eta(x, u)\right\rangle<0, \forall \xi_{i} \in \partial f_{i}(u), i \in K .
$$

For $\alpha(x, u)>0$, we have

$$
\left\langle\sum_{i=1}^{k} \bar{\lambda}_{i} \xi_{i}, \eta(x, u)\right\rangle<0, \forall \xi_{i} \in \partial f_{i}(u), i \in K .
$$

Since $(u, \bar{\lambda}, \bar{\mu}) \in M \quad$ is a feasible solution for (MD), there exist $\xi_{i} \in \partial f_{i}(u), i \in K$ and $\zeta_{j} \in \partial g_{j}(u), j \in M$ such that

$$
\sum_{i=1}^{k} \bar{\lambda}_{i} \xi_{i}+\sum_{j=1}^{m} \bar{\mu}_{j} \zeta_{j}=0
$$

whence

$$
\left\langle\sum_{i=1}^{k} \bar{\lambda}_{i} \xi_{i}, \eta(x, u)\right\rangle+\left\langle\sum_{j=1}^{m} \bar{\mu}_{j} \zeta_{j}, \eta(x, u)\right\rangle=0 .
$$

It follows from (35) that

$$
\left\langle\sum_{j=1}^{m} \bar{\mu}_{j} \zeta_{j}, \eta(x, u)\right\rangle>0
$$

For $\beta(x, u)>0$ and $\tau \geqq 0$, which yields

$$
\beta(x, u)\left\langle\sum_{j=1}^{m} \bar{\mu}_{j} \zeta_{j}, \eta(x, u)\right\rangle+\tau\|\psi(x, u)\|^{\sigma}>0 .
$$

From hypothesis 1), it follows that

$$
-b_{1}(x, u) \varphi_{1}\left[\sum_{j=1}^{m} \bar{\mu}_{j} g_{j}(u)\right]>0 .
$$

whence

$$
b_{1}(x, u) \varphi_{1}\left[\sum_{j=1}^{m} \bar{\mu}_{j} g_{j}(u)\right]<0 .
$$

which contradicts (33). Then the result is true.

The following definition is needed in the proof of the strong duality theorem.

Definition 4.1. A point $u \in X$ is called a strict maximizer of order $\sigma$ for (MD) with respect to a nonlinear function $\psi: X \times X \rightarrow R^{n}$, if there exists a constant $\rho \in$ int $R_{+}^{k}$ such that

$$
F(u)+\rho\|\psi(x, u)\|^{\sigma} \geqq F(x), \forall x \in X .
$$

Theorem 4.3. (strong duality) Assume that $\bar{x} \in X_{0}$ is a strict minimizer of order $\sigma$ with respect to $\psi$ for (MP), also there exist $\bar{\lambda} \geq 0, \sum_{i=1}^{k} \bar{\lambda}_{i}=1$ and $\bar{\mu} \geqq 0$, such that $0 \in \sum_{i=1}^{k} \bar{\lambda}_{i} \partial f_{i}(\bar{x})+\sum_{j=1}^{m} \bar{\mu}_{j} \partial g_{j}(\bar{x})$ and $\sum_{j=1}^{m} \bar{\mu}_{j} g_{j}(\bar{x})=0$. Fur- 
thermore, if all the hypothesis of Theorem 4.1 are satisfied for all feasible solutions of (MP) and (MD), then $(\bar{x}, \bar{\lambda}, \bar{\mu})$ is a strict maximizer of order $\sigma$ for (MD) with respect to $\psi$.

Proof: The hypothesis implies that $(\bar{x}, \bar{\lambda}, \bar{\mu})$ is a feasible solution of (MD). By Theorem 4.1, for any feasible $(y, \lambda, \mu)$ of (MD), we have

$$
f(\bar{x}) \geqq F(y)-\rho\|\psi(\bar{x}, y)\|^{\sigma} .
$$

That is

$$
f_{i}(\bar{x}) \geqq f_{i}(y)+\sum_{j=1}^{m} \mu_{j} g_{j}(y)-\rho_{i}\|\psi(\bar{x}, y)\|^{\sigma}, i \in K .
$$

Using $\sum_{j=1}^{m} \bar{\mu}_{j} g_{j}(\bar{x})=0$, which yields

$$
f_{i}(\bar{x})+\sum_{j=1}^{m} \bar{\mu}_{j} g_{j}(\bar{x})+\rho_{i}\|\psi(\bar{x}, y)\|^{\sigma} \geqq f_{i}(y)+\sum_{j=1}^{m} \mu_{j} g_{j}(y), i \in K .
$$

whence

$$
F(\bar{x})+\rho\|\psi(\bar{x}, y)\|^{\sigma} \geqq F(y) .
$$

Thus $(\bar{x}, \bar{\lambda}, \bar{\mu})$ is a strict maximizer of order $\sigma$ for (MD) with respect to $\psi$.

\section{Conclusion}

In this paper, we have defined a class of new generalized functions. By using the new functions, we have presented a sufficient optimality condition and Wolfe type duality results for a nondifferentiable multiobjective problem. The present results can be further generalized for other programming problems.

\section{Acknowledgements}

This work is supported by Scientific Research Program Funded by Shaanxi Provincial Education Department (Program No. 15JK1456); Natural Science Foundation of Shaanxi Province of China (Program No. 2017JM1041).

\section{Conflicts of Interest}

The authors declare no conflicts of interest regarding the publication of this paper.

\section{References}

[1] Clarke, F.H. (1983) Nonsmooth Optimization. John Wiley \& Son, Hoboken.

[2] Kim, D.S. and Bae, K.D. (2009) Optimality Conditions and Duality for a Class of Nondifferentiable Multiobjective Programming Problems. Taiwanese Journal of Mathematics, 13, 789-804. https://doi.org/10.11650/twjm/1500405403

[3] Agarwal, R.P., Ahmad, I., Zusain, H. and Jayswal, A. (2010) Optimality and Duality in Nonsmooth Multiobjective Optimization Involving V-Type I Invex Functions. Journal of Inequalities and Applications, 2010, Article ID: 898626. 
https://doi.org/10.1155/2010/898626

[4] Jayswal, A. (2010) On Sufficiency and Duality in Multiobjective Programming Problem under Generalized $\alpha$-Type I Univexity. Journal of Global Optimization, 46, 207-216. https://doi.org/10.1007/s10898-009-9418-y

[5] Antczak, T. (2012) Proper Efficiency Conditions and Duality Results for Nonsmooth Vector Optimization in Banach Spaces under $(\varphi, \rho)$-Invexity. Nonlinear Analysis. Theory, Methods \& Applications, 75, 3107-3121. https://doi.org/10.1016/j.na.2011.12.009

[6] Long, X.J. (2011) Optimality Conditions and Duality for a Class of Nondifferentiable Multiobjective Fractional Programming Problems with $(C, \alpha, \rho, d)$-Convexity. Journal of Optimization Theory and Applications, 148, 197-208. https://doi.org/10.1007/s10957-010-9740-Z

[7] An, G. and Gao, X.Y. (2013) On Sufficiency in Multiobjective Fractional Programming Problems. Journal of Computational and Theoretical Nanoscience, 10, 2943-2948. https://doi.org/10.1166/jctn.2013.3306

[8] Zhen, Y.C. and Gao, X.Y. (2016) Sufficiency and Duality for Multiobjective Programming under New Invexity. Mathematical Problems in Engineering, 2016, Article ID: 8462602. https://doi.org/10.1155/2016/8462602

[9] Upadhyay, B.B. and Mishra, S.K. (2015) Nonsmooth Semi-Infinite Minmax Programming Involving Generalized $(\varphi, \rho)$-Invexity. Journal of Systems Science and Complexity, 28, 857-875. https://doi.org/10.1007/s11424-015-2096-6

[10] Yan, C.L. and Feng, B.C. (2015) Sufficiency and Duality for Nonsmooth Multiobjective Programming Problems Involving Generalized $(\varphi, \rho)$-V-Type I Functions. Journal of Mathematical Modelling and Algorithms in Operations Research, 14, 159-172. https://doi.org/10.1007/s10852-014-9264-X

[11] Bae, K.D. and Kim, D.S. (2013) Optimality and Duality for Nonsmooth Multiobjective Optimization Problems. Journal of Inequalities and Applications, 2013, 554. https://doi.org/10.1186/1029-242X-2013-554

[12] Ahmad, I. and Al-Homidan, S. (2015) Sufficiency and Duality in Nondifferentiable Multiobjective Programming Involving Higher Order Strong Invexity. Journal of Inequalities and Applications, 2015, 309.

https://doi.org/10.1186/s13660-015-0819-9 